\title{
The effects of early paternal depression on children's development
}

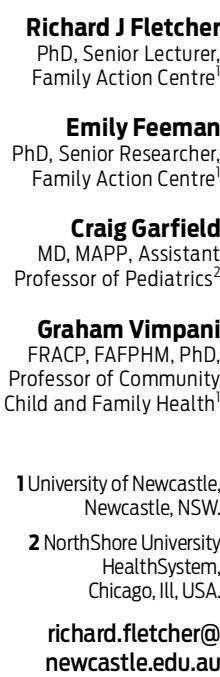

MJA 2011; 195: 685-689

doi: $10.5694 /$ mjall.10192

Online first 7/12/11 nfants depend on parents to develop their cognitive and emotional capacities. Internationally, maternal postnatal depression has been shown to adversely affect child wellbeing and development, so much so that maternal screening is standard in many countries. In Australia, the National Perinatal Depression Initiative (NPDI) is underway to provide universal screening and follow-up support for women assessed as being at risk of or experiencing perinatal depression. ${ }^{1}$ Assessments of the impact of fathers' depression on early child development have relied on cross-sectional studies ${ }^{2}$ and fathers are not currently targeted for screening or treatment in the NPDI.

However, more recently, evidence suggesting a causal relationship between fathers' postnatal depression and children's disrupted development has emerged. A United Kingdom study of 13351 families assessed fathers' depression using the Edinburgh Postnatal Depression Scale (the Edinburgh Scale) at 8 weeks after birth, and children's behaviour using maternal reports on the Rutter revised preschool scales at 3.5 years. ${ }^{3}$ Fathers' scores above 12 on the Edinburgh Scale were strongly associated with an increased risk of high total problems scores on the Rutter scales (odds ratio [OR], 2.19; 95\% CI, 1.55-3.08) and with high scores on the three subscales indicating problems with emotional regulation (eg, often unhappy ), conduct (eg, often lies or cheats) and hyperactivity (eg, constantly fidgeting). After controlling for social class, degree of education and maternal depression, only the association between fathers' postnatal depression and children's later conduct problems (OR, 1.73; 95\% CI, 1.06-2.85) and hyperactivity (OR, 1.96; 95\% CI, 1.12-3.43) remained. However, when the scores for boys and girls were considered separately, fathers' depression was associated with raised levels of problems for boys but not for girls (mothers' depression affected both boys and girls).

\begin{abstract}
Objective: To examine the effects of paternal depression during children's first year on their wellbeing at 4-5 years of age using a large, representative sample of Australian families.

Design, setting and participants: Prospective study of Australian families from 2004 to 2008. Two-biological-parent families $(n=2620)$ from the Longitudinal Study of Australian Children were included if depression measures were available for both parents in 2004, behavioural and developmental measures were available for children in 2008, and the families had not separated by 2008.
\end{abstract}

Main outcome measure: Child scores on the Strengths and Difficulties Questionnaire and on a set of Derived Outcome Indices, measured when the child was $4-5$ years old. Logistic regression modelling was used to investigate how early paternal depression in 2004 influenced child outcomes 4 years later.

Results: Early paternal depression was a significant predictor of a range of poorer child outcomes (odds ratio [OR] for behavioural difficulties, 3.34 [95\% Cl, 3.063.65]; OR for a low development and wellbeing score, 2.70 [95\% Cl, 2.44-2.98]). These effects remained significant after controlling for both early maternal depression and later paternal depression (adjusted OR for behavioural difficulties, 1.93 [95\% Cl, 1.75-2.14]; OR for a low development and wellbeing score, 1.65 [95\% $\mathrm{Cl}, 1.48-1.85])$.

Conclusions: Depression in fathers during the first year of a child's life can have a detrimental impact on their child's behaviour, and social and emotional development at the point of school entry, in addition to and uniquely compared with depression in mothers. Early intervention to identify and address the mental health needs of fathers is required for the benefit of fathers, children and families.

Thus, in the UK context, children, especially boys, of postnatally depressed fathers are at increased risk of behavioural problems.

The aim of the present study was to examine the effects of early paternal depression during children's first year on their wellbeing at $4-5$ years of age, using a large, representative sample of Australian families. We hypothesised that, after controlling for early maternal depression, children whose fathers had signs of early paternal depression would show poorer emotional and behavioural outcomes compared with children whose fathers had not shown early depressive symptoms. Our work extends that of previous studies by using multiple child outcome measures, thus allowing for a more complete understanding of the long-term effects of early paternal depression on child outcomes.

\section{Method}

\section{Sample}

Our study sample was drawn from the Longitudinal Study of Australian
Children (LSAC). The LSAC was initiated by and is funded by the Australian Government Department of Families, Community Services and Indigenous Affairs, and includes two representative samples of Australian children (see Soloff ${ }^{4}$ for methodological details). The LSAC collects data on children's early development, health, education and living circumstances. ${ }^{4}$ In this study, we used a confidentialised dataset made available to Australian academic researchers. We focused on the cohort of children aged 3-19 months ( $n=5104$; familylevel response rate, $64 \%$ ) at the first wave of data collection (2004), 2-3 years old at the second wave (2006), and $4-5$ years old in the third wave (2008). In order to ensure a similar level of exposure to paternal presence, we limited our sample to two-biological-parent families that were still intact 4 years after the initial wave of data collection $(n=2620)$.

\section{Measures}

The LSAC contains the shortened version of the Kessler Psychological Distress Scale (K6)..$^{5}$ All parents were assessed on the six items of the K6 
1 Early parental depressive symptoms and Strengths and Difficulties Questionnaire (SDQ) scores for children aged $4-5$ years

\begin{tabular}{|c|c|c|c|c|c|}
\hline & \multicolumn{2}{|c|}{ Proportion with high SDQ scores } & \multicolumn{3}{|c|}{ Odds ratio $(95 \% \mathrm{Cl})$} \\
\hline & Depressed & Not depressed & Crude & Adjusted* & Adjusted $^{\dagger}$ \\
\hline \multicolumn{6}{|l|}{ Maternal depression } \\
\hline Prosocial $^{\ddagger}$ & $10.2 \%$ & $12.4 \%$ & $0.76(0.68-0.86)$ & $0.69(0.61-0.78)$ & $0.75(0.66-0.84)$ \\
\hline Hyperactivity & $15.3 \%$ & $11.4 \%$ & $1.66(1.51-1.82)$ & $1.44(1.30-1.58)$ & $1.48(1.35-1.63)$ \\
\hline Emotional & $20.3 \%$ & $17.2 \%$ & $1.25(1.14-1.37)$ & $1.14(1.04-1.25)$ & $1.18(1.08-1.29)$ \\
\hline Peer problems & $32.2 \%$ & $17.5 \%$ & $2.96(2.75-3.19)$ & $2.73(2.53-2.94)$ & $2.81(2.60-3.03)$ \\
\hline Conduct & $35.6 \%$ & $18.6 \%$ & $2.38(2.20-2.57)$ & $2.16(2.00-2.34)$ & $2.18(2.02-2.36)$ \\
\hline Total difficulties & $23.7 \%$ & $10.7 \%$ & $2.48(2.28-2.70)$ & $2.06(1.88-2.25)$ & $2.14(1.96-2.33)$ \\
\hline \multicolumn{6}{|l|}{ Paternal depression } \\
\hline Prosocial ${ }^{\ddagger}$ & $21.4 \%$ & $12.2 \%$ & $2.10(1.90-2.31)$ & $2.16(1.96-2.39)$ & $2.65(2.39-2.94)$ \\
\hline Hyperactivity & $26.2 \%$ & $11.3 \%$ & $2.47(2.25-2.71)$ & $1.95(1.77-2.14)$ & $1.41(1.26-1.58)$ \\
\hline Emotional & $26.2 \%$ & $17.1 \%$ & $1.62(1.48-1.78)$ & $1.39(1.27-1.53)$ & $1.10(0.98-1.22)$ \\
\hline Peer problems & $26.2 \%$ & $17.7 \%$ & $1.64(1.50-1.80)$ & $1.16(1.06-1.27)$ & $0.74(0.66-0.83)$ \\
\hline Conduct & $26.2 \%$ & $18.9 \%$ & $1.69(1.55-1.85)$ & $1.22(1.11-1.34)$ & $0.92(0.83-1.02)$ \\
\hline Total difficulties & $28.6 \%$ & $10.7 \%$ & $3.34(3.06-3.65)$ & $2.30(2.10-2.52)$ & $1.93(1.75-2.14)$ \\
\hline
\end{tabular}

*Adjusted for socioeconomic position, maternal education, and depression in the other parent. †Adjusted for socioeconomic position, maternal education, depression in the other parent, and later paternal depression. $¥$ For prosocial behaviour, bottom $10 \%$ of scores were used to indicate a poor outcome. (rated 0-4, maximum score of 24), which asks respondents how they have been feeling over the past 30 days. Scores of 8-12 have been shown to predict moderate psychiatric symptoms, while scores $>12$ have been shown to indicate clinically significant depressive symptoms. ${ }^{6}$ The $\mathrm{K} 6$ has been shown to have good internal consistency and concordance with other, longer measures of depression (eg, the Diagnostic and statistical manual of mental disorders, 4 th edition $)^{6}$ and has been used effectively in Australian populations to measure mood and anxiety disorders. ${ }^{7}$ The $\mathrm{K} 6$ has also been used as an indicator of depression in a wide range of studies $^{8-10}$ and is recommended as a screening measure for depression in population mental health surveys. ${ }^{11}$ We created a dichotomous variable identifying parents who had symptoms consistent with depression during the perinatal period (ie, $\mathrm{K} 6$ scores $>12$; Wave 1 data collection in 2004).

\section{Child outcome measures}

The Strengths and Difficulties Questionnaire (SDQ) is a widely used and validated measure of a child's emotional and behavioural functioning, with reasonable internal consistency (Cronbach's $\alpha, 0.73$ ), and retest stability. ${ }^{12}$ In LSAC, it was completed by the primary parent ( $98 \%$ mothers). The SDQ was developed from the
Rutter scales and consists of five subscales (hyperactivity, emotional problems, conduct problems, peer problems and prosocial behaviour scores) and a total difficulties score. ${ }^{13}$ Following Ramchandani and colleagues, ${ }^{3}$ we dichotomised scores at the top $10 \%$ to indicate a high score on each of the five difficulty scores and at the bottom $10 \%$ to indicate a low prosocial score. SDQ difficulties scores above the 90th percentile indicate problem behaviours and are associated with a substantially increased risk of developing a psychiatric disorder. $^{12}$

Derived Outcome Indices (DOIs) were developed specifically for the LSAC as aggregate indicators of children's overall development and wellbeing. ${ }^{13}$ They cover physical, learning and social/emotional outcomes, and can be combined to give an overall outcome index. The DOIs are derived from a multistage standardisation process that cumulates in a standardised score for each domain (mean = 100; SD, 10). Following the recommendation by Sanson, ${ }^{13}$ we dichotomised scores at the bottom $15 \%$ to indicate poor child outcomes.

\section{Statistical analysis}

Analyses were conducted with PASW Statistics, version 18 (SPSS Inc, Chicago, Ill, USA). As per Ramchandani et $\mathrm{al}^{3}{ }^{3}$ we calculated the prevalence of depression in both mothers and fathers and then conducted LSACpopulation-weighted logistic regression analyses to examine the associations between paternal depression and our child outcome measures, controlling for socioeconomic position, maternal education, and maternal depression. Maternal and paternal education were significantly correlated and maternal education data was more complete, so we chose to replicate the procedure outlined by Ramchandani et $\mathrm{al}^{3}$ by using only maternal education in each of our models. Additional models controlling for the effects of later paternal depression (measured in 2006) using a dummy coded indicator variable were run in order to ensure that the effects of paternal depression were specific to the perinatal period. Additionally, we tested for statistical interactions between parental depression and the sex of the child, and report separate odds ratios for boys and girls for each outcome measure.

Our study did not require ethics approval, as it was an analysis of a deidentified publicly available dataset.

\section{Results}

The prevalence of early depression in this sample was similar to the rates for the full LSAC sample, with $2.6 \%$ of mothers and $1.3 \%$ of fathers having K6 scores $>12$. Maternal and paternal scores on the $\mathrm{K} 6$ were significantly correlated $(r=0.19 ; P<0.001)$.

\section{Strengths and Difficulties Questionnaire}

Early paternal depression was associated with an increased risk of a high SDQ total difficulties score (OR, 3.34; 95\% CI, 3.06-3.65). It was also associated with all the other subscales. After controlling for socioeconomic position, education and maternal depression, these associations remained significant (Box 1). Early maternal depression was also associated with an increased risk of a high SDQ total difficulties score (OR, 2.48; 95\% CI, 2.28-2.70). Maternal depression was also associated with all the SDQ subscales and these associations remained significant after controlling for socioeconomic position, education and paternal depression. When we 
controlled for later paternal depression, prosocial behaviour, hyperactivity, and SDQ total difficulties scores were still significantly associated with early paternal depression. Associations with maternal depression remained stable (Box 1). Early paternal depression was found to have a larger effect on boys' hyperactivity and prosocial scores than on those of girls, but a larger effect on girls' emotional, conduct and total difficulties scores. Additionally, early maternal depression was found to have a larger effect on boys' prosocial and on girls' conduct scores (Box 2).

\section{Derived Outcome Indices}

Early paternal depression was associated with an increased risk of a low DOI overall outcome score (OR, 2.70; 95\% CI, 2.44-2.98) and was associated with lower scores on all of the DOI subscales. After controlling for socioeconomic position, education, and maternal depression, only the associations with social/emotional outcome and overall outcome scores remained significant (Box 3). Early maternal depression was associated with an increased risk of a low overall outcome score (OR, 2.87; 95\% CI, 2.63-3.14) and with each of the DOI subscales. After controlling for socioeconomic position, education, and paternal depression, these associations remained significant. When we controlled for later paternal depression, early paternal depression was associated with an increased risk of lower overall outcome and social/ emotional outcome scores and maternal depression was associated with all DOI scales (Box 3). All the associations between paternal depression and DOI scores were stronger for girls than boys. The associations between maternal depression and social/emotional outcome scores were stronger for girls than boys, but the associations with learning scores and overall outcome scores were stronger for boys (Box 4).

\section{Discussion}

Our results confirm our hypothesis that children whose fathers reported depressive symptoms when they were infants would have poorer outcomes at 4-5 years of age than children

2 Differential effects of parental depression on boys' and girls' Strengths and Difficulties Questionnaire scores

\begin{tabular}{|c|c|c|c|c|}
\hline & \multicolumn{2}{|c|}{ Adjusted odds ratio* $(95 \% \mathrm{Cl})$} & \multirow[b]{2}{*}{$\begin{array}{l}\text { Likelihood ratio test for } \\
\text { interaction }{ }^{\dagger}\end{array}$} & \multirow[b]{2}{*}{$P$} \\
\hline & Boys & Girls & & \\
\hline \multicolumn{5}{|c|}{ Maternal depression } \\
\hline Prosocial & $1.12(0.78-1.28)$ & $0.24(0.1-0.33)$ & 92 & $<0.001$ \\
\hline Hyperactivity & $1.60(1.41-1.81)$ & $1.42(1.22-1.66)$ & 1 & $>0.05$ \\
\hline Emotional & $1.11(0.97-1.27)$ & $1.21(1.07-1.36)$ & 2 & $>0.05$ \\
\hline Peer problems & $2.91(2.62-3.24)$ & $2.84(2.54-3.16)$ & $<1$ & $>0.05$ \\
\hline Conduct & $1.94(1.73-2.17)$ & $2.46(2.21-2.74)$ & 10 & $<0.01$ \\
\hline Total difficulties & $2.22(1.97-2.52)$ & $2.12(1.87-2.41)$ & $<1$ & $>0.05$ \\
\hline \multicolumn{5}{|l|}{ Paternal depression } \\
\hline Prosocial & $3.50(3.02-4.06)$ & $2.54(2.16-2.99)$ & 187 & $<0.001$ \\
\hline Hyperactivity & $1.89(1.62-2.21)$ & $1.26(1.06-1.50)$ & 169 & $<0.001$ \\
\hline Emotional & $0.57(0.46-0.71)$ & $1.44(1.26-1.64)$ & 146 & $<0.001$ \\
\hline Peer problems & $0.67(0.56-0.80)$ & $0.88(0.76-1.02)$ & 32 & $<0.001$ \\
\hline Conduct & $0.66(0.55-0.79)$ & $1.16(1.02-1.33)$ & 109 & $<0.001$ \\
\hline Total difficulties & $1.09(0.91-1.31)$ & $2.98(2.62-3.39)$ & 305 & $<0.001$ \\
\hline
\end{tabular}

*Adjusted for socioeconomic position, maternal education, depression in the other parent, and later paternal depression. $\dagger$ By sex.

\section{Early parental depressive symptoms and Derived Outcome Indices (DOI) scores for children aged 4-5 years}

\begin{tabular}{|c|c|c|c|c|c|}
\hline & \multicolumn{2}{|c|}{ Proportion with low DOI scores } & \multicolumn{3}{|c|}{ Odds ratio $(95 \% \mathrm{Cl})$} \\
\hline & Depressed & Not depressed & Crude & Adjusted* & Adjusted $^{\dagger}$ \\
\hline \multicolumn{6}{|l|}{ Maternal depression } \\
\hline Physical & $27.1 \%$ & $10.8 \%$ & $3.59(3.32-3.89)$ & $3.45(3.18-3.74)$ & $3.44(3.18-3.73)$ \\
\hline Learning & $8.5 \%$ & $9.0 \%$ & $0.87(0.77-0.99)$ & $0.73(0.64-0.83)$ & $0.77(0.68-0.88)$ \\
\hline Social/emotional & $18.6 \%$ & $8.2 \%$ & $2.76(2.52-3.02)$ & $2.30(2.10-2.52)$ & $2.44(2.22-2.67)$ \\
\hline Overall & $16.9 \%$ & $7.8 \%$ & $2.87(2.63-3.14)$ & $2.45(2.23-2.68)$ & $2.59(2.36-2.84)$ \\
\hline \multicolumn{6}{|l|}{ Paternal depression } \\
\hline Physical & $14.3 \%$ & $11.1 \%$ & $1.42(1.27-1.59)$ & $1.06(0.95-1.19)$ & $1.04(0.92-1.18)$ \\
\hline Learning & $17.1 \%$ & $8.9 \%$ & $1.90(1.71-2.11)$ & $1.11(0.98-1.24)$ & $0.98(0.86-1.11)$ \\
\hline Social/emotional & $23.8 \%$ & $8.1 \%$ & $3.37(3.07-3.70)$ & $2.36(2.14-2.60)$ & $1.75(1.57-1.96)$ \\
\hline Overall & $17.1 \%$ & $7.9 \%$ & $2.70(2.44-2.98)$ & $1.42(1.28-1.58)$ & $1.65(1.48-1.85)$ \\
\hline
\end{tabular}

*Adjusted for socioeconomic position, maternal education, and depression in the other parent. $\nmid$ Adjusted for socioeconomic position, maternal education, depression in the other parent, and later paternal depression.

whose fathers did not have early depressive symptoms. Specifically, children whose fathers had early depressive symptoms were more likely to score above the 90th percentile on the SDQ total difficulties, hyperactivity, emotional, peer problems and conduct scales, and below the 10th percentile on the prosocial scale. These associations remained significant after controlling for demographic characteristics, early maternal depression and, with the exceptions of emotional, peer and conduct problems, later paternal depression. A number of these associations were found to interact with the sex of the child. For example, early paternal depression was found to be more strongly associated with hyperactivity problems in boys than girls, but was more strongly associated with emotional problems in girls than boys. With regard to the more generalised developmental outcomes, we found that early paternal depression was associated with poorer social/emotional and overall outcomes in children, and that this effect was stronger for girls than boys. These findings contrast with those of other studies, ${ }^{3,14}$ which found a greater effect of early paternal depression on boys' outcomes.

Although our results confirm the importance of maternal depression on child outcomes, when focusing on paternal depression our results support previous research, ${ }^{3,14,15}$ and demonstrate that early paternal 
depression is also important for child outcomes regardless of whether maternal depressive symptoms are present or not. In addition, the effects of maternal and paternal depression vary with the sex of the child, suggesting that maternal and paternal depression differ in the way that they affect child development.

Previous findings that depressed fathers are less likely to participate in physical play with their young children, ${ }^{16}$ an activity purported to assist children with their regulation of behaviours, ${ }^{17,18}$ suggests a possible area for future investigations.

Our findings also showed differences by sex across all child outcome measures for fathers, but only a few outcomes showed sex differences for mothers. Other areas of research have also shown stronger sex differences for fathers than mothers. For example, it has been suggested that fathers engage in more gender socialisation than mothers across a wide range of aspects of children's social and emotional development. ${ }^{19}$ Fathers have been shown to engage in warmer and more structured interactions and to be more sensitive with their daughters than with their sons, whereas mothers showed no such differentiation. ${ }^{20}$ If, as the research suggests, fathers, but not mothers, differentially interact with their sons and daughters, then finding sex differences in the impact of early paternal, but not maternal, depression on child outcomes should be expected.

Although the underlying mechanism remains unclear, our findings

\begin{tabular}{|c|c|c|c|c|}
\hline & \multicolumn{2}{|c|}{ Adjusted odds ratio* $(95 \% \mathrm{Cl})$} & \multirow[b]{2}{*}{$\begin{array}{l}\text { Likelihood ratio test } \\
\text { for interaction }{ }^{\dagger}\end{array}$} & \multirow[b]{2}{*}{$P$} \\
\hline & Boys & Girls & & \\
\hline \multicolumn{5}{|c|}{ Maternal depression } \\
\hline Physical & $3.66(3.27-4.09)$ & $3.29(2.93-3.70)$ & 2 & $>0.05$ \\
\hline Learning & $1.26(1.08-1.46)$ & $0.40(0.30-0.54)$ & 42 & $<0.001$ \\
\hline Social/emotional & $2.08(1.82-2.38)$ & $3.16(2.78-3.59)$ & 41 & $<0.001$ \\
\hline Overall & $3.77(3.34-4.25)$ & $1.82(1.57-2.11)$ & 40 & $<0.001$ \\
\hline \multicolumn{5}{|l|}{ Paternal depression } \\
\hline Physical & $0.21(0.16-0.29)$ & $2.45(2.14-2.81)$ & 183 & $<0.001$ \\
\hline Learning & $0.63(0.51-0.78)$ & $2.08(1.77-2.46)$ & 293 & $<0.001$ \\
\hline Social/emotional & $0.72(0.57-0.90)$ & $3.36(2.93-3.87)$ & 405 & $<0.001$ \\
\hline Overall & $0.72(0.59-0.89)$ & $3.06(2.66-3.51)$ & 145 & $<0.001$ \\
\hline
\end{tabular}

may be limited. Compared with the full LSAC sample, parents in our sample were more highly educated, had a higher weekly income, and a higher percentage were in full-time employment. The rate of depressive symptoms among both mothers and fathers in this sample is lower than that reported in other Australian samples. ${ }^{24,25}$ It is possible that non-completers may have been more depressed, but if this were the case, the association between early paternal depression and later child outcomes would likely be strengthened. Future research should examine the effects of early paternal depression in these populations and ideally would replace the self-report measure of depression (the K6) with formal psychiatric diagnoses.

Another limitation of our study was the variability in the age range of the infants at Wave 1 of LSAC data collection, with underrepresentation of recently born children in the sample. As such, the results from our study need to be interpreted as being associated with early paternal depression, not paternal postnatal depression, which is usually defined as occurring in the first few months after a child's birth. ${ }^{3}$ Nevertheless, given the dearth of research on the long-term effect of paternal depression on children, our findings are notable.

Our study has demonstrated that self-reported depression in fathers during the first year of a child's life can have a detrimental impact on their child's behaviour, and social and emotional development at the point of school entry. Furthermore, these effects are independent from mothers' symptomatology, supporting the notion that fathers play an important role in a child's development that is independent of the mother's role. Although the causal pathways leading from early paternal depression to poor child outcomes have yet to be explicated, our study adds to the literature identifying this as a discrete and important issue for families and children. Early intervention to identify and address the mental health needs of fathers may be required for the benefit of fathers, children and families.

Acknowledgements: This study was supported by a Strategic Initiatives research grant funded by the Faculty of Health, University of Newcastle. The researchers are independent from the funder. We used data from the 
LSAC, which was conducted in partnership between the Department of Families, Housing Community Services and Indigenous Affairs (FaHSCIA), the Australian Institute of Family Studies (AIFS) and the Australian Bureau of Statistics (ABS). The findings reported in this article are those of the authors and should not be attributed to FaHCSIA, the AIFS or the ABS.

Competing interests: No relevant disclosures.

Received 23Feb 2011, accepted 27 Jul 2011.

1 Australian Health Ministers' Advisory Council. Framework for the National Perinatal Depression Initiative 2008-09 to 2012-13. http:// www.ahmac.gov.au/site/home.aspx (accessed Feb 2011).

2 Kane P, Garber J. The relations among depression in fathers, children's psychopathology, and father-child conflict: a meta-analysis. Clin Psychol Rev 2004; 24: 339-360.

3 Ramchandani P, Stein A, Evans J, et al. Paternal depression in the postnatal period and child development: a prospective population study. Lancet 2005; 365: 2201-2205.

4 Soloff C, Lawrence D, Johnstone R. Sample design. Melbourne: Australian Institute of Family Studies, 2005. (Longitudinal Study of Australian Children Technical Paper No. 1.)

5 Kessler RC, Andrews G, Colpe LJ, et al. Short screening scales to monitor population prevalences and trends in non-specific psychological distress. Psychol Med 2002; 32: 959-976.

6 Kessler RC, Green JG, Gruber MJ, et al. Screening for serious mental illness in the general population with the $\mathrm{K} 6$ screening scale: results from the WHO World Mental Health (WMH) survey initiative. Int JMethods Psychiatr Res 2010; 19: 4-22

7 Furukawa TA, Kessler RC, Slade T et al. The performance of the $\mathrm{K} 6$ and $\mathrm{K} 10$ screening scales for psychological distress in the Australian National Survey of Mental Health and WellBeing. Psychol Med 2003; 33: 357-362.

8 Baggaley RF, Ganaba R, Filippi V, et al. Detecting depression after pregnancy: the validity of the $\mathrm{K} 10$ and K6 in Burkina Faso. Trop Med Int Health 2007; 12: 1225-1229.

9 Holden L, Scuffham P, Hilton M, et al. Psychological distress is associated with a range of high-priority health conditions affecting working Australians. Aust N Z J Public Health 2010; 34: 304-310

10 Vigod SN, Kurdyak PA, Stewart DE, et al. Depressive symptoms as a determinant of breast and cervical cancer screening in women: a population-based study in Ontario, Canada. Arch Womens Ment Health 2011; 14: 159-168.

11 Cairney J, Veldhuizen S, Wade TJ, et al. Evaluation of 2 measures of psychological distress as screeners for depression in the general population. Can J Psychiatry 2007; 52: 111-120.

12 Goodman R. Psychometric properties of the Strengths and Difficulties Questionnaire (SDQ) $J$ Am Acad Child Adolesc Psychiatry 2001; 40: 1337-1345.

13 Sanson A, Misson S, Wake M, et al. Summarising children's wellbeing: the LSAC Outcome Index. Melbourne: Australian Institute of Family Studies, 2005. (Longitudinal Study of Australian Children Technical Paper No. 2.)

14 Ramchandani P, Stein A, O'Connor TG, et al. Depression in men in the postnatal period and later child psychopathology: a population cohort study.J Am Acad Child Adolesc Psychiatry 2008; 47: 390-398.

15 Weinfield NS, Ingerski L, Moreau SC. Maternal and paternal depressive symptoms as predictors of toddler adjustment. J Child Fam Stud 2009; 18 : 39-47.
16 Paulson JF, Dauber S, Leiferman JA. Individual and combined effects of postpartum depression on parenting behavior in mothers and fathers. Pediatrics 2006; 118: 659-668.

17 Carson J, Burks V, Parke RD. Parent-child physical play: determinants and consequences. In MacDonald K, editor. Parent-child play: descriptions and implications. Albany, NY: State University of New York Press, 1993.

18 Flanders JL, Simard M, Paquette D, et al. Roughand-tumble play and the development of physical aggression and emotion regulation: a five-year follow-up study. J Fam Violence 2010; 25: 357-367.

19 Chaplin TA, Cole PM, Zahn-Waxler C. Parental socialization of emotion expression: gender differences and relations to child adjustment. Emotion 2005; 5: 80-88.

20 Feldman R, Klein PS. Toddlers' self-regulated compliance to mothers, caregivers, and fathers: implications for theories of socialization. Dev Psychol 2003; 39: 680-692.

21 Fletcher R, Matthey S, Marley CG. Addressing depression and anxiety among new fathers. Med J Aust 2006; 185: 461-463.

22 Condon J. What about dad? Psychosocial and mental health issues for new fathers. Aust Fam Physician 2006; 35: 690-692.

23 Wilhelm KA. Men and depression. Aust Fam Physician 2009; 38: 102-106.

24 Paulson JF, Bazemore SD. Prenatal and postpartum depression in fathers and its association with maternal depression: A metaanalysis. JAMA 2010; 303: 1961-1969.

25 Buist AE, Austin MPV, Hayes B, et al. Postnata mental health of women giving birth in Australia 2002-2004: findings from the beyondblue National Postnatal Depression Program. Aust NZ JPsychiatry 2008; 42: 66-73. 